\title{
Sonocrystallization yields monoclinic paracetamol with significantly improved compaction behavior
}

\author{
Dejan-Krešimir Bučar, ${ }^{*}$ James A. Elliott, ${ }^{*}$ Mark D. Eddleston, Jeremy K. Cockcroft and
}

William Jones

\begin{abstract}
Ultrasound-assisted crystallization (sonocrystallization) was used to prepare a mixture of nano- and micrometer-sized crystals of the monoclinic form of paracetamol - a widely used analgesic known for its particularly problematic mechanical behavior under compression (i.e. poor tabletability). The nano- and microsized crystals yielded a powder which exhibits elastic moduli and bulk cohesions that are significantly higher than those observed in samples consisting of macro-sized crystals, thus leading to enhanced tabletability without the use of excipients, particle coating, salt- or cocrystal formation. Experimental compaction and finite element analysis were utilized to rationalize the significantly improved compaction behavior of the monoclinic form of paracetamol.
\end{abstract}

The current demand for organic nanomaterials ${ }^{[1-3]}$ has triggered the development of fabrication methods for organic nanocrystals based on single ${ }^{[4-10]}$ and multiple components (i.e. cocrystals). ${ }^{[11-14]}$ Recent pharmaceutical and biomedical research has shown that molecular nanocrystals are highly valuable in the context of drug delivery, biolabeling and biosensing. ${ }^{[15-19]}$ Nano-sizing of molecular crystals has also become a particularly popular approach in drug development of poorly soluble pharmaceutical agents ${ }^{[20]}$ and is mainly used to enhance the solubility and bioavailability of the drug candidate. ${ }^{[21-23]}$ Surprisingly, other applications of molecular crystals, despite their unique physicochemical properties, have been relatively unexplored in biomedical (and other) sciences. We show in this contribution that nano-sized crystals can significantly improve the mechanical properties of a crystalline pharmaceutical agent via sonocrystallization, ${ }^{[24-26]}$ i.e. the use of ultrasound to facilitate crystallization. ${ }^{[27-28]}$

Paracetamol (pca, Fig. 1), also known as acetaminophen, is an exceptionally popular analgesic and antipyretic that achieves

[*] Dr. Jeremy Karl Cockcroft and Dr. Dejan-Krešimir Bučar Department of Chemistry

University College London

20 Gordon Street, London WC1H 0AJ, United Kingdom

E-mail: d.bucar@ucl.ac.uk

Dr. James A. Elliott

Department of Materials Science and Metallurgy,

University of Cambridge

27 Charles Babbage Road, Cambridge CB3 OFS, United Kingdom

E-mail: jae1001@cam.ac.uk

Dr. Mark D. Eddleston and Prof. William Jones

Department of Chemistry,

University of Cambridge

Lensfield Road, Cambridge CB2 3QZ, United Kingdom annual sales of more than 1 billion USD in the United States alone. ${ }^{[29]}$ Pca exhibits two well-known polymorphs - the monoclinic ${ }^{[30]}$ form I and the orthorhombic ${ }^{[31]}$ form II. An elusive and metastable polymorph, form III, has only recently been structurally characterized. ${ }^{[32]}$ The thermodynamically stable form I exhibits poor tabletability under compression. ${ }^{[33-35]}$ The orthorhombic form II, on the other hand, displays significantly better compaction properties, ${ }^{[33,36]}$ but is thermodynamically less stable $^{[37-38]}$ and, thus, not ideal for drug formulation and commercialization. The poor tabletability of form $\mathbf{I}$ is attributed to its crystal structure. In particular, form I is based on corrugated hydrogen-bonded layers of pca molecules and lacks the presence of flat molecular layers that can promote the formation of microscopic slip planes capable of rendering the crystalline solid plastic and easier to compact (Fig. 1a) ${ }^{[33]}$ Form II, on the other hand, exhibits slip planes comprised of flat hydrogenbonded pca layers ${ }^{[31]}$ that promote better tabletability (Fig. 1b).

Pca is generally formulated using the poorly compactible monoclinic form I along with up to 25 wt $\%$ of excipients to reduce occurrence of defective tablets, such as capping, lamination or chipping. ${ }^{[39]}$ The poorly compactible crystals of from I can also be coated with hydroxypropyl cellulose to improve their behavior under compression. ${ }^{[0]}$ Alternatively, pca can be formulated as a salt ${ }^{[41]}$ or cocrystal ${ }^{[42-43]}$ in order to facilitate the assembly of crystal structures that contain slip planes that result in improved tabletability.
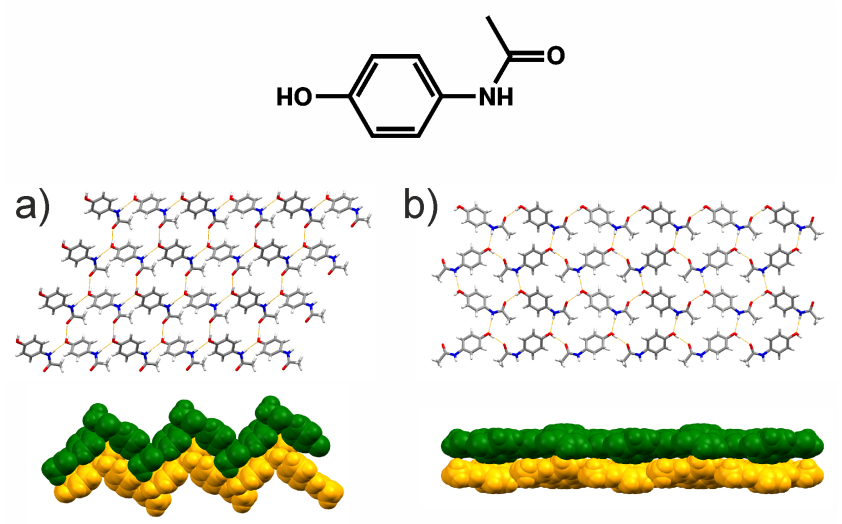

Figure 1. Chemical structure of pca (top) and the crystal structures of a) form I (monoclinic, stable) and b) form II (orthorhombic, metastable) featuring corrugated and flat molecular sheets found in their respective crystal structures.

A recent study has shown that freeze-drying of wateracetone solutions of pca leads to the formation of micro- 

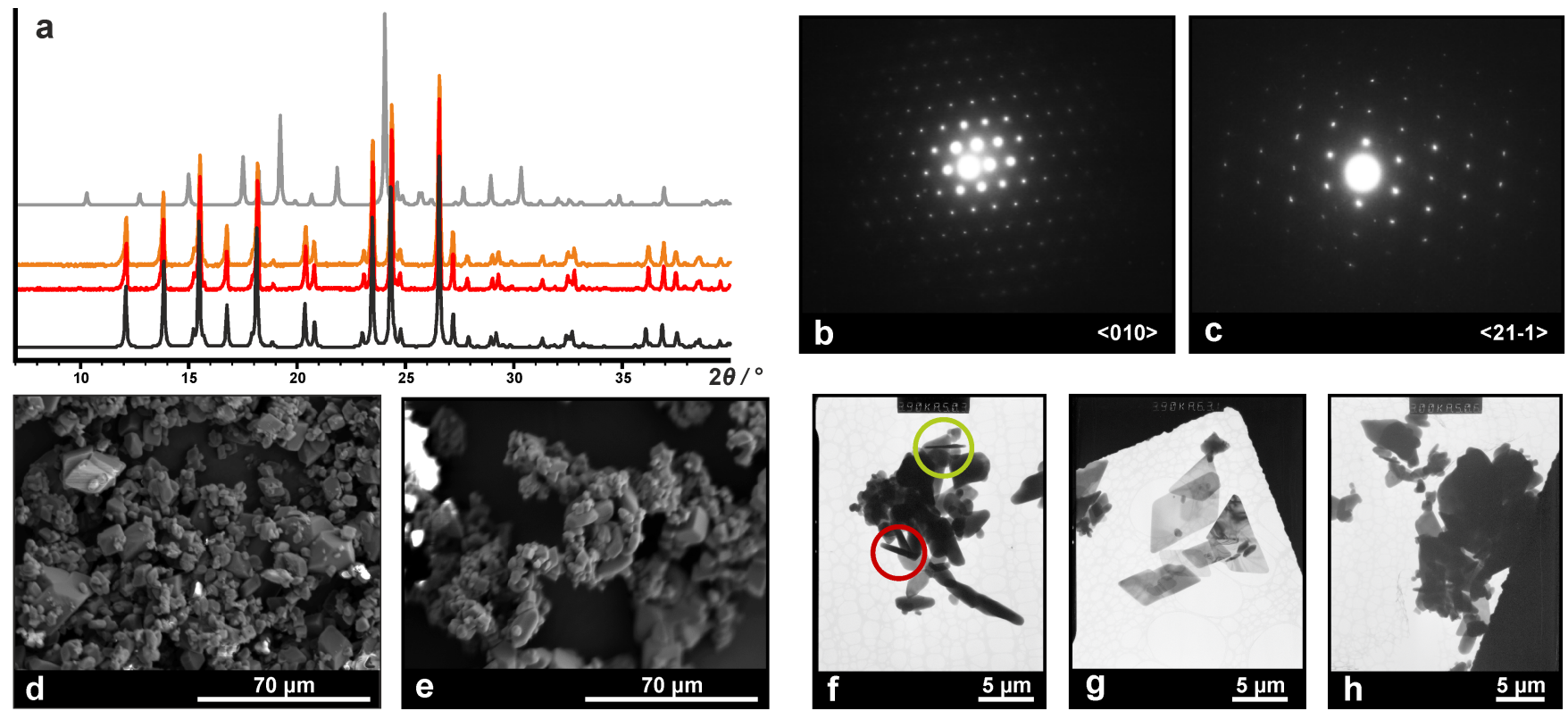

Figure 2. Crystallographic characterization of the sonocrystallized bulk of form I: a) PXRD patterns of pca (black - calculated form I, red - sonocrystallized form I, orange - sonocrystallized form I after compaction, grey - calculated form II) and b,c) SAED patterns obtained from individual nano-dimensional single crystals are consistent with the formation of form I (b: <010> zone axis, c: <21-1> zone axis). The SEM and TEM images shown in d, e and f-h (respectively) depict sonocrystallized nano- and micro-particles of form I exhibiting broad particle size distributions. The green and red circles in micrograph (f) highlight nanocrystalline plates with a measured thickness of $220-230 \mathrm{~nm}$ (green) and $470-590 \mathrm{~nm}$ (red).

crystals of form I with enhanced compaction properties. ${ }^{[44]}$ Specifically, it was demonstrated that tablets based on submicrometer-sized crystals exhibit a high compressive strength of $40 \mathrm{MPa}$ (axially) and about $6 \mathrm{MPa}$ (diametrally), as well as a low porosity of $6 \%$. Such tablets, however, need to be prepared using a compaction pressure of $345 \mathrm{MPa}$ - which exceeds those typically used in industrial tableting processes (i.e. in the range of $100-150 \mathrm{MPa}$ ) to limit damage to dies and tooling. ${ }^{[45]}$ Furthermore, it was suggested that the freeze-drying method is not economically viable for inexpensive drugs such as pca. ${ }^{[44]}$

We show in this article that time- and cost-efficient ultrasound-assisted crystallization yields submicrometer- and micrometer-sized crystals of form I, which were shown to exhibit elastic moduli and bulk cohesions that are significantly higher than those observed in macrocrystalline form I, giving rise to a remarkable increase in tabletability at compaction pressures that are typically used in current industrial processes. We also reveal that mechanochemically prepared nano-crystals (using liquidassisted grinding as a production method) exhibit narrower particle size distributions and are not as tabletable as the sonocrystallized solid, thus suggesting that particle size distribution possibly plays a significant role in the improvement of the compaction behavior of form $\mathbf{I}$.

Combined sonication and fast precipitation afforded the formation of a physical mixture of nano- and micro-crystals of form I. In a typical sonocrystallization experiment, pca was dissolved in a minimal amount of ethanol to obtain a solution that was immediately injected through a syringe filter into hexane while exposed to ultrasound. The suspension was sonicated for $1 \mathrm{~min}$, filtered and dried at room temperature. The obtained solid was analyzed by X-ray powder diffraction (PXRD), selected area electron diffraction (SAED), scanning-electron microscopy (SEM) and transmission electron microscopy (TEM). Details of the synthesis and analysis are provided in the Supporting Information (SI) document.

A PXRD analysis of the bulk material, in addition to SAED measurements of individual nanocrystals, revealed that pca crystallizes as form I (Fig. 2a-c). The presence of the orthorhombic form II could not be detected via PXRD and SAED. A thorough inspection of the SEM and TEM micrographs indicated that the sonocrystallized sample exhibits a broad particle-size distribution. Specifically, the micrographs revealed the presence of aggregated nano- and micrometer-sized crystals with plate and prism morphologies and prism in the bulk (Fig. 2d-h, and Fig. S3-S6 in the SI document). The micrographs also showed that the crystals are uniform in shape, with particle sizes ranging from $\sim 0.14 \mu \mathrm{m}$ to $\sim 40 \mu \mathrm{m}$ in length. The thickness of the crystal plates was estimated based on TEM images and to be as low as $\sim 200 \mathrm{~nm}$. A particle-size distribution analysis was attempted using dynamic light scattering (DLS) and powder $X$ ray diffraction (PXRD). The DLS results indicated that the nanoand microcrystals form aggregates with an average diameter of $60 \mu \mathrm{m}$. Although crystal agglomeration can be prevented by the use of surfactants, we have not considered the use of surfactants to characterize the sonocrystallized solid, as they are known to alter crystal size and morphology. ${ }^{[46]}$ The PXRD data did not reveal any measurable peak broadening that could be attributed $o$ the presence of nanocrystals in the bulk. This observation is consistent with the sample's broad particle-size 
a)

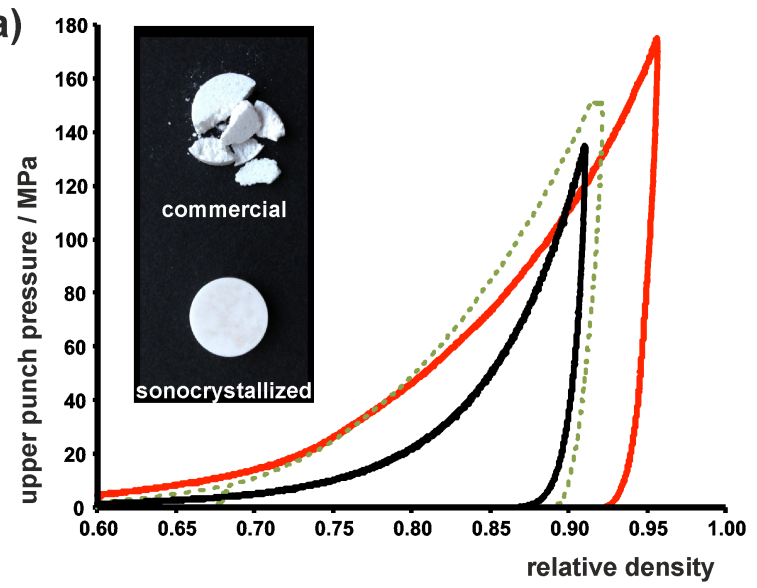

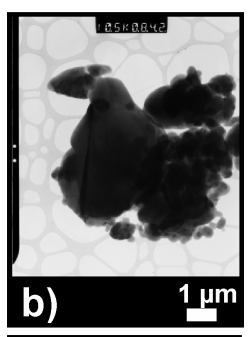

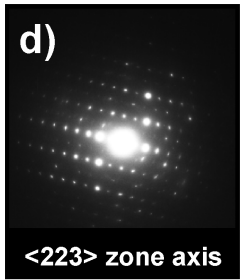

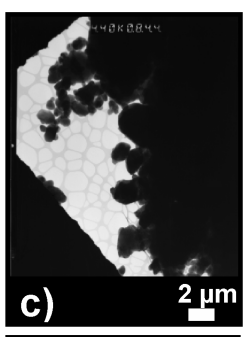

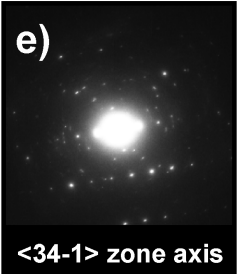

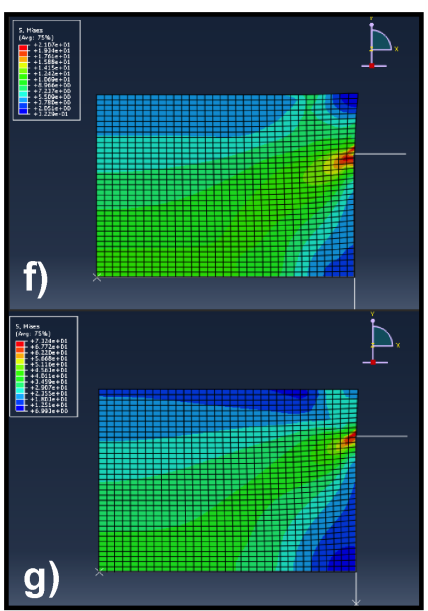

Figure 3. a) Comparison of stress-density curves for macrocrystalline (black), sonocrystallised (red), mechanochemically prepared (dotted green) form I. Relative density refers to the powder density relative to the density of the crystal structure of form I $\left(\rho=1.263 \mathrm{~g} \mathrm{~cm}{ }^{3}\right.$ ) (inset: tablets composed of commercial and sonocrystallized form I after compaction); b,c) TEM micrographs showing that the compacted nano- and microcrystals of form I shattered into smaller particles; d,e) SAED patterns obtained from individual nano-dimensional compacted single crystals reveal that compaction has lead to an increase in crystal defect, but no phase transition; $\mathrm{f}, \mathrm{g}$ ) stress distributions calculated from axisymmetric finite element simulations of cylindrical tablets comprised of macrocrystalline form I and nanocrystalline form I during ejection from die (the top of die wall is indicated by a horizontal white line on far right-hand side of the figures, and left-hand side is the axis of die, about which the simulation is cylindrically symmetrical).

distribution and the presence of micrometer-sized crystals in the sonocrystallized bulk material.

To evaluate the compaction behavior of commercial (i.e. macrocrystalline) and sonochemically (i.e. nano-/microcrystalline) prepared batches of form I, we utilized a hydraulic uniaxial compaction simulator with an instrumented die (Huxley Bertram), allowing simultaneous measurement of axial and radial stresses and, ultimately, stress-density curves for the studied materials. Relevant parameters relating to the mechanical properties of the solids are shown in Table 1, wherein they are also compared to those of Avice ${ }^{\beta}$ PH101 (a pharmaceutical-grade microcrystalline cellulose commonly used as binder to hold the ingredients in a tablet together).

Table 1. Material parameters for powders characterized by compaction simulator using DPC constitutive model.

\begin{tabular}{lcc}
\hline material & $\begin{array}{c}\text { Young's modulus } \\
(E / \mathrm{MPa})\end{array}$ & $\begin{array}{c}\text { cohesion } \\
(d / \mathrm{MPa})\end{array}$ \\
\hline Avice $^{\circledR}(\mathrm{PH} 101)$ & 3700 & 4.2 \\
pca (commercial) & 2612 & 0.1 \\
pca (sonocrystallized) & 6365 & 3.4 \\
\hline
\end{tabular}

As expected, it was observed that tablets formed from the commercial pca sample crumbled on ejection from the die. ${ }^{[33-35]}$ In contrast, the tablets made from sonocrystallized pca retained their sharp edges (Fig. 3a, inset). A comparison of the stressdensity curves for sonocrystallized and commercial pca is shown in Fig. 3a. It can be seen that, during unloading phase, the sonocrystallized form I obtained a higher relative density than the macrocrystalline form at the same applied pressure, indicating that more plastic deformation had taken place. The final relative densities of tablets after ejection from die were $0.922(7.8 \%$ porosity) and $0.866(13.4 \%$ porosity), respectively, indicating that the tablet formed from sonocrystallized material is more than $40 \%$ less porous than the tablet formed from macrocrystalline powder under same conditions.

Compaction simulations using the Drucker-Prager cap constitutive model ${ }^{[47-48]}$ revealed that particles of sonocrystallized form I exhibit higher elastic moduli than the macrocrystalline one (Table 1). The higher elastic modulus suggests that sonocrystallized form I should have an inferior ability to plastically deform, thus leading to poor compaction properties. The improved tabletability of sonocrystallized form $\mathbf{I}$ is, however, attributed to its greater compactability (i.e. higher relative density under given load) and the greater amount of plastic work dissipated during compaction. Both of these effects feasibly arise indirectly from the nano-sized particles that exhibit a greater total contact area between particles thus increasing their cohesion and packing density compared to macrocrystalline sample. Despite an apparent increase in elastic modulus of the sonocrystallized form I relative to macrocrystalline form (by a factor of 2.4, see Table 1), the vastly increased cohesion (by a factor of 34, see Table 1) and extra plastic work during deformation mean that tablet fracture due to elastic recovery on decompression is less likely in the tablet made from sonocrystallized powder.

A TEM study on the tableted powder of the sonocrystallized batch of form I was also carried out to determine whether a polymorphic phase transition has occurred that could lead to the improved compaction behavior. For this purpose, we halved a tablet, and investigated only the solid that "crumbled" off the halved tablet and has, thus, not been in contact with the utilized tools. A PXRD measurement showed that the solid still consists exclusively of form I crystals (Fig. 2a). In addition, TEM 
micrographs (Fig. 3b-e) showed that the investigated crystals correspond to form I. The observed crystals are considerably smaller in size (i.e. $\sim 1 \mu \mathrm{m}$ along all crystal axes) (Fig. 3b,c), thus indicating that the nano- and microcrystals cracked into smaller particles during the compaction process. This is further evidenced by SAED experiments that revealed that the crystals exhibited a significantly higher degree of mosaicity (Fig. 3d,e), as compared to those prior to compaction. Moreover, a PXRD trace of the compacted material revealed broadening of the diffraction peaks that is consistent with a decrease of the material's particle size (average particle-size: $\sim 1.5 \mu \mathrm{m}$, see SI document).

To assess the behavior of pca under high pressure, axisymmetric finite element simulations of cylindrical tablets during ejection from die were used to calculate the stress distributions (Mises deviatoric stress, in $\mathrm{MPa}$ ) within the compacted commercial and sonocrystallized solid. Figures $3 f$ and $3 \mathrm{~g}$ show the stress distribution for commercial (i.e. macrocrystalline) and sonocrystallized (i.e. nano-/micro-sized) pca at the same point during simulation, and shows that the stress distribution is more anisotropic for the commercial solid, especially near the edges of the tablet. Based on previous findings for tableting simulations using pharmaceutical excipients, ${ }^{[4]}$ this would suggest that tablets made from macrocrystalline pca would be more likely to chip or fail mechanically during the ejection phase.

The above-described results raised the question of whether the mechanical properties of form I can be further improved by preparing even smaller crystals that are also uniform in size. To evaluate this possibility, we turned to liquid-assisted grinding ${ }^{[49-}$ 50] - a mechanochemical method used to generate high-shear and impact forces for the making of uniform nano-dimensional crystals. ${ }^{[51]}$ In a typical experiment, a macro-crystalline batch of form I was milled in the presence of hexane. PXRD was used to identify the milled pca sample as form I, while PXRD and SEM were used to define the sample as nano-crystalline and more uniform (as compared to sonocrystallized pca) with an average particle size of $1 \mu \mathrm{m}$. Details of the PXRD particle-size analyses are provided in the SI document.

The compaction studies showed that tablets made from milled pca crumbled on ejection from the die, as seen in the case of commercial pca (see the SI document). Similarly to the sonocrystallized solid, the milled material consisted exclusively of form I crystals that have decreased in size upon exposure to the high pressures (average particle size: $0.9 \mu \mathrm{m}$, see SI document). Furthermore, the stress-density curve of the milled sample exhibited a relative density of 0.891 ( $10.9 \%$ porosity), which is higher than that of the commercial sample $(0.866$, $13.4 \%$ porosity), but lower than that of sonocrystallized pca (0.922, $7.8 \%$ porosity). It is interesting to compare the behavior of the sonocrystallized and milled samples, both of which contain appreciable amounts of nano-sized crystals (Fig. 2 and 3; also, see the SI document). Despite this similarity, Figure 3 shows that the load curve for milled form I (dotted green line) differs significantly from both macrocrystalline and sonocrystallized samples (black and red, respectively). In particular, the sonocrystallized sample reaches a higher maximum relative density (i.e. lower porosity) for loads exceeding $40 \mathrm{MPa}$, and has a larger hysteresis on unloading, indicating a greater amount of mechanical work is lost to plastic deformation or fracture of particles on compaction. The increased propensity to plastic deformation of nano-sized crystals is known for metallic systems, ${ }^{[52]}$ and may also be a contributing factor to the improved tabletability of the sonocrystallized and milled form $\mathbf{I}$ over the commercial macrocrystalline solid. The origin of the poorer compaction properties of the milled solid when compared to those of the sonocrystallized solid remains, at this point, to be fully understood.

To conclude, we have shown that pca form I, an analgesic and antipyretic known for its poor tabletability, can be crystallized using a combination of ultrasonication and fast precipitation to obtain a mixture of nano- and macrocrystals that display significantly improved tabletability at pressures that are typically used in current industrial processes, and in the absence of any excipients. The superior tabletability is attributed to the solid' $s$ enhanced ability to plastically deform and the cohesive interactions between its particles that are significantly higher than those observed in the macrocrystalline samples. We have also shown that milled form I (consisting of smaller uniform particles) displays compaction properties that are inferior to those observed for the sonocrystallized material, thus suggests the possibility that a broader particle size distribution promotes the tabletability of pca. We believe that the presented results demonstrate the utility of sonocrystallization in the preparation of pharmaceutically relevant molecular nano/macrocrystals with superior properties. Studies are underway to evaluate the impact of particle size and distribution on the mechanical properties of other organic solids obtained using bottom-up (e.g. sonocrystallization) and top-down (e.g. milling) methods.

\section{Acknowledgements}

DKB acknowledges the Royal Society for a Newton International Fellowship and University College London for an UCL Excellence Fellowship. DKB and WJ thank the Isaac Newton Trust (Trinity College, University of Cambridge) for funding. JAE would like to thank M. Bennett, M. House and W. Bertram of Huxley Bertram for the use of their compaction simulator. WJ and MDE acknowledge support by the Interreg IV "2 Mers Seas Zeeën" cross-border cooperation program 2007-2013.

Keywords: molecular nanocrystals • sonocrystallization • ultrasound $\cdot$ powder compaction $\cdot$ polymorphism

[1] Single Organic Nanoparticles, Springer Berlin Heidelberg, 2003.

[2] Nanomaterials Chemistry: Recent Developments and New Directions, Wiley-VCH Verlag GmbH \& Co. KGaA., Weinheim, 2007.

[3] Organic Nanomaterials: Synthesis, Characterization, and Device Applications, Wiley-VCH Verlag GmbH \& Co. KGaA, Weinheim, 2013.

[4] T. Asahi, T. Sugiyama, H. Masuhara, Acc. Chem. Res 2008, 41, 17901798.

[5] A. A. Date, V. B. Patravale, Curr. Opin. Colloid In. 2004, 9, 222-235.

[6] K. Baba, H. Kasai, S. Okada, H. Oikawa, H. Nakanishi, Opt. Mater. 2003, 21, 591-594. 
[7] V. Monnier, N. Sanz, E. Botzung-Appert, M. Bacia, A. Ibanez, J. Mater. Sci. 2006, 16, 1401-1409.

[8] E. Kwon, H. Oikawa, H. Kasai, H. Nakanishi, Cryst. Growth Des. 2007, 7, 600-602.

[9] J. R. G. Sander, D.-K. Bučar, J. Baltrusaitis, L. R. MacGillivray, J. Am. Chem. Soc. 2012, 134, 6900-6903.

[10] B. Sinha, R. H. Müller, J. P. Möschwitzer, Int. J. Pharm. 2013, 453 , 126-141.

[11] D.-K. Bučar, L. R. MacGillivray, J. Am. Chem. Soc. 2006, 129, 32-33.

[12] J. R. G. Sander, D.-K. Bučar, R. F. Henry, G. G. Z. Zhang, L. R. MacGillivray, Angew. Chem. Int. Ed. 2010, 49, 7284-7288.

[13] C. Karunatilaka, D.-K. Bučar, L. R. Ditzler, T. Friščić, D. C. Swenson, L. R. MacGillivray, A. V. Tivanski, Angew. Chem. Int. Ed. 2011, 50, 86428646.

[14] D. Yan, D.-K. Bučar, A. Delori, B. Patel, G. O. Lloyd, W. Jones, X. Duan, Chem. Eur. J. 2013, 19, 8213-8219.

[15] H. Kasai, T. Murakami, Y. Ikuta, Y. Koseki, K. Baba, H. Oikawa, H. Nakanishi, M. Okada, M. Shoji, M. Ueda, H. Imahori, M. Hashida Angew. Chem. Int. Ed. 2012, 51, 10315-10318.

[16] Y. Ikuta, Y. Koseki, T. Murakami, M. Ueda, H. Oikawa, H. Kasai, Chem. Lett. 2013, 42, 900-901.

[17] K. Baba, H. E. Pudavar, I. Roy, T. Y. Ohulchanskyy, Y. Chen, R. K. Pandey, P. N. Prasad, Mol. Pharm. 2007, 4, 289-297.

[18] S. Fery-Forgues, Nanoscale 2013, 5, 8428-8442.

[19] X. Yang, T.-C. Ong, V. K. Michaelis, S. Heng, J. Huang, R. G. Griffin, A. S. Myerson, CrystEngComm 2014.

[20] J. P. Möschwitzer, Int. J. Pharm. 2013, 453, 142-156.

[21] E. Merisko-Liversidge, G. G. Liversidge, E. R. Cooper, Eur. J. Pharm. Sci. 2003, 18, 113-120

[22] E. M. Merisko-Liversidge, G. G. Liversidge, Toxicol. Pathol. 2008, 36, 43-48.

[23] M. Wang, G. C. Rutledge, A. S. Myerson, B. L. Trout, J. Pharm. Sci. 2012, 101, 1178-1188.

[24] M. D. Luque de Castro, F. Priego-Capote, Ultrason. Sonochem. 2007, 14, 717-724

[25] H. Xu, B. W. Zeiger, K. S. Suslick, Chem. Soc. Rev. 2013, 42, 25552567.

[26] J. R. G. Sander, B. W. Zeiger, K. S. Suslick, Ultrason.Sonochem. 2014, 21, 1908-1915.

[27] K. S. Suslick, Science 1990, 247, 1439-1445.

[28] K. S. Suslick, S. J. Doktycz, Vol. 1 (Ed.: T. J. Mason), JAI Press, New York, 1990, pp. 197-230.

[29] W. M. Lee, Hepatology 2004, 40, 6-9.
[30] M. Haisa, S. Kashino, R. Kawai, H. Maeda, Acta Crystallogr. B 1976, 32, 1283-1285.

[31] M. Haisa, S. Kashino, H. Maeda, Acta Crystallogr. B 1974, 30, 25102512.

[32] M.-A. Perrin, M. A. Neumann, H. Elmaleh, L. Zaske, Chem. Commun. 2009, 3181-3183.

[33] G. Nichols, C. S. Frampton, J. Pharm. Sci. 1998, 87, 684-693.

[34] M. Duberg, C. Nyström, Powder Technol. 1986, 46, 67-75.

[35] I. Krycer, D. G. Pope, J. A. Hersey, Journal of Pharmacy and Pharmacology 1982, 34, 802-804.

[36] E. Joiris, P. Martino, C. Berneron, A.-M. Guyot-Hermann, J.-C. Guyot, Pharm. Res. 1998, 15, 1122-1130.

[37] K. Kachrimanis, K. Fucke, M. Noisternig, B. Siebenhaar, U. Griesser, Pharm. Res. 2008, 25, 1440-1449.

[38] T. Beyer, G. M. Day, S. L. Price, J. Am. Chem. Soc. 2001, 123, 50865094.

[39] J. S. M. Garr, M. H. Rubinstein, Int. J. Pharm. 1991, 72, 117-122.

[40] C. C. Sun, L. Shi, Pharm. Res. 2011, 28, 3248-3255.

[41] S. R. Perumalla, L. Shi, C. C. Sun, CrystEngComm 2012, 14, 23892390.

[42] S. Karki, T. Friščić, L. Fábián, P. R. Laity, G. M. Day, W. Jones, Adv. Mater. 2009, 21, 3905-3909.

[43] Y. Maeno, T. Fukami, M. Kawahata, K. Yamaguchi, T. Tagami, T. Ozeki, T. Suzuki, K. Tomono, Int. J. Pharm. 2014, 473, 179-186.

[44] A. Ogienko, E. Boldyreva, A. Yu Manakov, V. Boldyrev, A. Yunoshev, A. Ogienko, S. Myz, A. Ancharov, A. Achkasov, T. Drebushchak, Pharm. Res. 2011, 28, 3116-3127

[45] G. Ragnarsson, in Pharmaceutical Powder Compaction Technology (Eds.: G. Alderborn, C. Nyström), Marcel Dekkers Inc., New York, 1996, pp. 77-97.

[46] J. P. Canselier, J. Disper. Sci. Technol. 1993, 14, 625-644.

[47] L. H. Han, J. A. Elliott, A. C. Bentham, A. Mills, G. E. Amidon, B. C. Hancock, Int. J. Solids Struct. 2008, 45, 3088-3106.

[48] C. Y. Wu, O. M. Ruddy, A. C. Bentham, B. C. Hancock, S. M. Best, J. A. Elliott, Powder Technol. 2005, 152, 107-117.

[49] D.-K. Bučar, S. Filip, M. Arhangelskis, G. O. Lloyd, W. Jones, CrystEngComm 2013

[50] T. Friščić, W. Jones, Cryst. Growth Des. 2009, 9, 1621-1637.

[51] G. D. Wang, F. P. Mallet, F. Ricard, J. Y. Y. Heng, Current Opinion in Chemical Engineering 2012, 1, 102-107.

[52] H. Zheng, A. Cao, C. R. Weinberger, J. Y. Huang, K. Du, J. Wang, Y. Ma, Y. Xia, S. X. Mao, Nat. Commun. 2010, 1, 144. 


\section{COMMUNICATION}

Text for Table of Contents

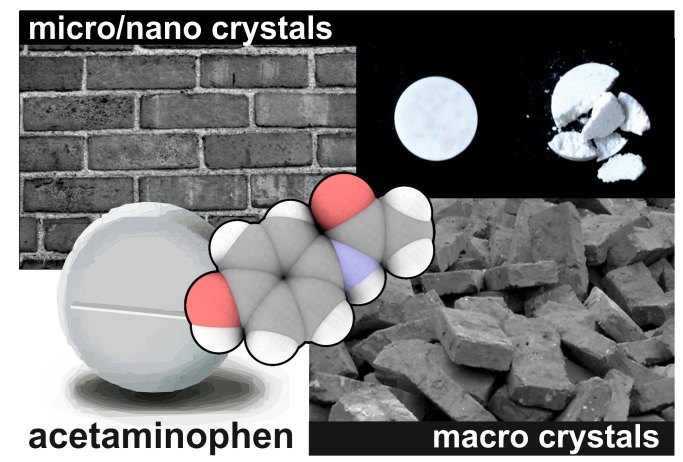

Dejan-Krešimir Bučar, ${ }^{*}$ James A. Elliott, * Mark D. Eddleston, Jeremy K. Cockcroft and William Jones

Page No. - Page No.

Sonocrystallization yields monoclinic paracetamol with significantly improved compaction behavior 